EPJ Web of Conferences 41, 02033 (2013)

DOI: $10.1051 /$ epjconf/20134102033

(C) Owned by the authors, published by EDP Sciences, 2013

\title{
Quantum-dynamical Modeling of the Rydberg to Valence Excited-State Internal Conversion in Cyclobutanone and Cyclopentanone
}

\author{
T. S. Kuhlman ${ }^{1}$, S. P. A. Sauer ${ }^{2}$, T. I. Sølling ${ }^{2}$, and K. B. Møller ${ }^{1}$ \\ ${ }^{1}$ Technical University of Denmark, Department of Chemistry, DK-2800 Kgs. Lyngby, Denmark \\ ${ }^{2}$ University of Copenhagen, Department of Chemistry, DK-2100, København Ø, Denmark
}

\begin{abstract}
In this paper we present 4-state, 5-dimensional Vibronic Coupling Hamiltonians for cyclobutanone and cyclopentanone. Wave packet calculations using these Hamiltonians reveal that for cyclobutanone the $(n, 3 \mathrm{~s})$ to $\left(n, \pi^{*}\right)$ internal conversion involves direct motion in nuclear modes coupling the two states leading to fast population transfer. For cyclopentanone, internal vibrational energy redistribution is a bottleneck for activating reactive nuclear modes leading to slower population transfer.
\end{abstract}

\section{Introduction}

Processes in excited states initiated by short laser pulses can exhibit significant non-ergodic behavior as exemplified by the cycloketones [1,2]. The direct time-scale for the $(n, 3 \mathrm{~s})$ to $\left(n, \pi^{*}\right)$ transition in these molecules exhibit such effects [2]. A perturbative approach, i.e., Fermi's golden rule, should be able to describe the rate of such a transition

$$
w_{m \leftarrow n}(E)=(2 \pi / \hbar)\left|\left\langle\Psi_{m}|V| \Psi_{n}\right\rangle\right|^{2} \rho_{m}(E) .
$$

Here $\rho_{m}(E)$ is the density of vibrational states of the final electronic state $\left|\Psi_{m}\right\rangle$ and $V$ is the perturbation coupling the two states. Contrary to the expectation of a rise in the rate of transition as $\rho_{m}(E)$ is increased, the exact opposite trend was observed for the cycloketones [2]. This observation points to a significant coordinate dependence of the coupling matrix element in Eq. 1. To investigate this, we present in here the construction of 4-state, 5-dimensional vibronic coupling Hamiltonians (VCHAM) [3] for cyclobutanone and cyclopentanone and their use in wave packet calculations to investigate the true dynamical nature of the $(n, 3 \mathrm{~s})$ to $\left(n, \pi^{*}\right)$ transition in these molecules.

\section{Computational Methods}

Wave packet calculations were performed using the Heidelberg Multi-configuration TimeDependent Hartree (MCTDH) code [4]. The VCHAMs were fitted on the basis of 1182 and $1273 a b$ initio points for cyclobutanone and cyclopentanone, respectively, with a locally modified version of the VCHFIT program distributed with the Heidelberg MCTDH code.

Quantum chemistry calculations were performed in Gaussian 03 [5], CFOUR [6], and Dalton [7] at the coupled-cluster singles and doubles (CCSD) [8,9] and equation of motion (EOM) or linear

This is an Open Access article distributed under the terms of the Creative Commons Attribution License 2.0, which permits unrestricted use, distribution, and reproduction in any medium, provided the original work is properly cited. 
response CCSD levels of theory. The cc-pVTZ basis set [10] was augmented by a 1s1p1d set of diffuse functions to describe the Rydberg states [11].

\section{The Vibronic Coupling Hamiltonian}

The VCHAM is given in dimensionless normal mode coordinates obtained at the ground state equilibrium geometry. A subset of modes were chosen such as to describe the significant changes between the equilibrium structure of the ground, $\left(n, \pi^{*}\right)$, and $(n, 3 \mathrm{~s})$ states; ring-pucker (labeled $v_{1} / v_{1}$ for cyclobutanone/cyclopentanone), carbonyl out-of-plane deformation $\left(v_{2} / v_{3}\right)$, symmetric C-CO-C stretch $\left(v_{7} / v_{8}\right)$, asymmetric C-CO-C stretch $\left(v_{12} / v_{16}\right)$, and $\mathrm{C}=\mathrm{O}$ stretch $\left(v_{21} / v_{28}\right)$.

Fig. 1 and Fig. 2 give examples of the fit between the parameterized VCHAMs and the ab initio data. For the ground state of cyclobutanone and cyclopentanone the RMSD between ab initio data and fit is 4.97 and $13.9 \mathrm{meV}$ respectively. For the three excited states the RMSD is $8.2 \mathrm{meV}$ and $4.28 \mathrm{meV}$ for cyclobutanone and cyclopentanone respectively.
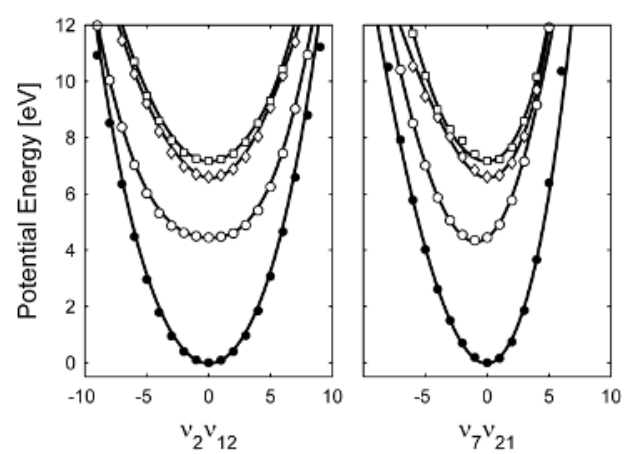

Fig. 1. Ab initio points (symbols) for the four lowest states of cyclobutanone along the mode diagonals $v_{2} v_{12}$ and $v_{7} v_{21}$ as well as the potential energy surfaces obtained from the fitted VCHAM (lines).
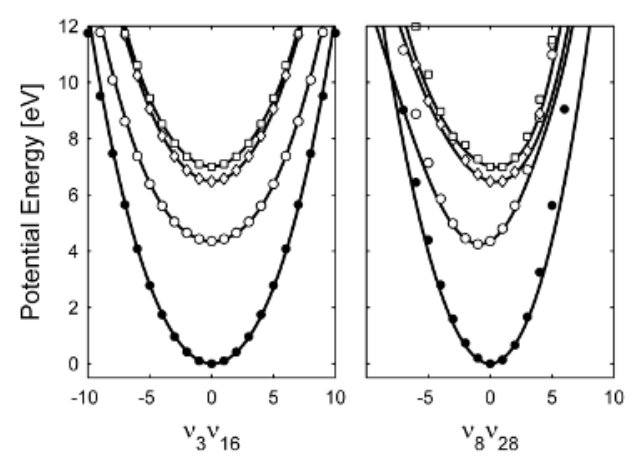

Fig. 2. Ab initio points (symbols) for the four lowest states of cyclopentanone along the mode diagonals $v_{3} v_{16}$ and $v_{8} v_{28}$ as well as the potential energy surfaces obtained from the fitted VCHAM (lines).

\section{Dynamics}

Although the potential energy surfaces of cyclobutanone and cyclopentanone are very similar for the five modes included in the VCHAMs as exemplified in Fig. 1 andFig. 2, very different time-scales for the transition between the initial $(n, 3 \mathrm{~s})$ and final $\left(n, \pi^{*}\right)$ state is observed. Fig. 3 shows the diabatic populations of the three excited states following excitation to the $(n, 3 \mathrm{~s})$ state. The decay of the $(n, 3 \mathrm{~s})$ population can for both molecules be adequately fitted by a decaying biexponential function with time-constants of $0.95 \mathrm{ps}$ and $6.32 \mathrm{ps}$ for cyclobutanone and $3.99 \mathrm{ps}$ and $81.5 \mathrm{ps}$ for cyclopentanone. The short time-constants are in good correspondence with the ones obtained from time-resolved mass spectrometry of 0.74 ps and 5.30 ps for cyclobutanone and cyclopentanone respectively [2].

A significant difference between the two molecules can be found in the symmetries of their excited states. For cyclobutanone, which belongs to the $\mathrm{C}_{\mathrm{s}}$ point group, both the $\left(n, \pi^{*}\right)$ and the $(n, 3 \mathrm{~s})$ state belong to the A" irreducible representation, and it is thus the Franck-Condon active modes $v_{1}$, $v_{2}, v_{7}$, and $v_{21}$ belonging to $A^{\prime}$, which are responsible for the linear vibronic coupling part of the perturbation operator $V$ of Eq. 1. As a consequence direct motion in the coupling modes occurs right after excitation to the $(n, 3 \mathrm{~s})$ state and population transfer is fast. On the other hand, for cyclopentanone, which belongs to the $\mathrm{C}_{2}$ point group, the $\left(n, \pi^{*}\right)$ state belongs to the A irreducible representation whereas the $(n, 3 \mathrm{~s})$ state belongs to $\mathrm{B}$. As a consequence it is the non-Franck-Condon 
active modes $v_{1}, v_{3}$, and $v_{16}$ belonging to $\mathrm{B}$, which are responsible the linear vibronic coupling. Motion in the coupling modes does therefore not occur until vibrational energy redistribution has transferred energy from the Franck-Condon modes $v_{8}$ and $v_{28}$ into the coupling modes - a process which is observed to take on the order of $10 \mathrm{ps}$. As a consequence population transfer is an in-direct process and slower than what is observed in the case of cyclobutanone.
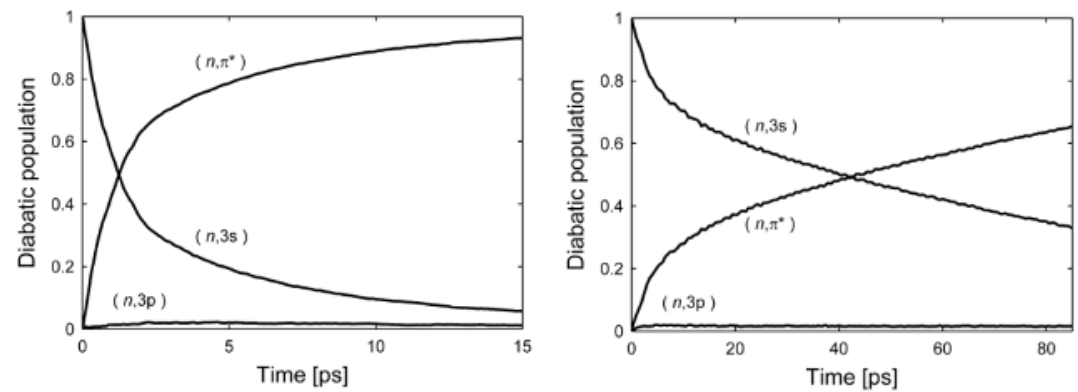

Fig. 3. Diabatic populations of the $\left(n, \pi^{*}\right),(n, 3 \mathrm{~s})$, and $(n, 3 \mathrm{p})$ states as a function of time following excitation to the $(n, 3 \mathrm{~s})$ state for cyclobutanone (left) and cyclopentanone (right).

\section{Conclusion}

Similar to experiment, wave packet calculations reveal that the $(n, 3 \mathrm{~s})$ to $\left(n, \pi^{*}\right)$ transition is faster in cyclobutanone compared to cyclopentanone. The difference of time-scales can be explained from the two different mechanisms of internal conversion; one direct where motion in the reactive coupling modes leads to a fast population transfer and one indirect where internal vibrational energy redistribution (IVR) from the non-reactive modes is a bottleneck for activating the reactive coupling modes. In the direct mechanism the transition takes place on a time-scale shorter than that of IVR and thus non-ergodicity prevails. In the indirect mechanism, IVR takes place on a timescale comparable to or slightly shorter than that of the transition and ergodicity somewhat prevails.

\section{References}

1. E. W.-G. Diau, J. L. Herek, Z. H. Kim, A. H. Zewail, Science 279, 847 (1998)

2. T. S. Kuhlman, T. I. Sølling, K. B. Møller, ChemPhysChem 13, 820 (2012)

3. L. S. Cederbaum, W. Domcke, H. Köppel, and W. von Niessen, Chem. Phys. 26, 169 (1977)

4. G. A. Worth, M. H. Beck, A. Jäckle, and H.-D. Meyer, The MCTDH Package, Version 8.2, (2000). H.-D. Meyer, Version 8.3 (2002), Version 8.4 (2007). See http://mctdh.uni-hd.de

5. M. J. Frisch et al., Gaussian 03, Revision E.01, Gaussian, Inc., Wallingford, CT, 2004.

6. J. F. Stanton et al., CFOUR, a quantum chemical program package, For the current version, see http://www.cfour.de

7. Dalton, a molecular electronic structure program, release 2.0 (2005), see http://daltonprogram.org

8. D. Mukherjee and P. K. Mukherjee, Chem. Phys. 39, 325 (1979)

9. H. Koch and P. Jørgensen, J. Chem. Phys. 93, 3333 (1990)

10. T. H. Dunning, J. Chem. Phys. 90, 1007 (1989)

11. B. O. Roos et al., Adv. Chem. Phys. 93, 219 (1996). 